\title{
Channel Simulation of Non-imaging Optical MIMO Communication
}

\author{
Jiao Feng, Liwei Ding, Yongjin Wang \\ Institute of Communication Technology, Nanjing University of Posts and Telecommunications, Jiangsu, China \\ Email: wangyj@njupt.edu.cn
}

Received 2013

\begin{abstract}
This paper reports a channel simulation of an indoor optical wireless multiple-input-multiple-output (MIMO) system with non-imaging receivers. The system consists of a $2 \times 2$ array of white light-emitting diodes (LEDs) and $2 \times 2$ array of PDs. An overview of the model specifications, channel impulse response and channel capacity are demonstrated in this paper. The distribution of the first reflection is analyzed. The effect of SNR and the location of receivers on non-imaging optical MIMO communications are investigated. In addition, by moving the receivers, the optimal location of the communication is found.
\end{abstract}

Keywords: MIMO; LEDs; Non-imaging; Channel Impulse Response; Channel Capacity

\section{Introduction}

Energy-saving is gradually becoming the key focus all over the world. LED devices have been widely applied to home and industry because of this trend. In addition to the function of illumination, the white LEDs can also be used for communication [1]. The main challenge of LEDs communications is the limited modulation bandwidth of sources, just several MHZ [2,3]. Many solutions are proposed in the past several years such as high efficient modulation schemes, RGB multi chip LED modules and so on. Recently, researchers pay more attention to the multiple-input-multiple-output (MIMO) technology which could provide parallel data transmission for the sake of achieving high data rates[4-8]. Many works are also done to explore the channel capacity. [9] investigates the MIMO channel capacity in correlated channels and [10] shows the channel capacity of the imaging receivers system. In this paper, an entirely possible non-imaging optical MIMO communications model is established to explore the design specifications, channel impulse response and distribution of the channel capacity. The rest of this paper is organized as follows, section 2 describes the specifications of the system we setup. Section 3 gives the channel impulse response. Section 4 shows the capacity of the model. Section 5 concludes the work before and analyses the future of the visible light communication.

\section{System Setup}

The MIMO system in this paper is implemented through intensity modulation and direct detection (IM/DD). The system consists of four transmitter units (LEDs). The arrangement of the LEDs in the room is shown in Figure 1. The room size is $5 \mathrm{~m} * 5 \mathrm{~m} * 2.5 \mathrm{~m}$. The LEDs are arranged in four corners of room. Table 1 shows the parameters of the system.

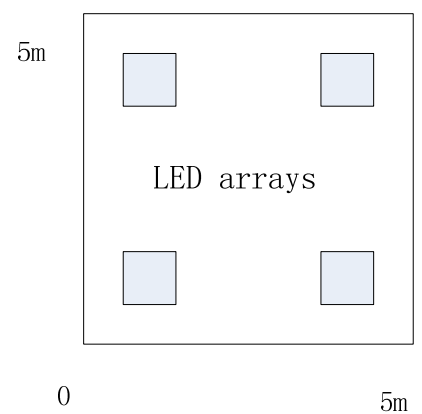

Figure 1. The distribution of the LEDs in the room.

Table 1. Parameters of MIMO model.

\begin{tabular}{lc}
\hline \multicolumn{1}{c}{ Parameters } & values \\
\hline Room size $(\mathrm{W} \times \mathrm{L} \times \mathrm{H})$ & $5 \mathrm{~m} \times 5 \mathrm{~m} \times 2.5 \mathrm{~m}$ \\
power of single LED & $5 \mathrm{w}$ \\
FOV at a receiver(half-angle) & $60^{\circ}$ \\
Detector physical area of a PD & $0.15 * 0.15$ \\
Center luminous intensity & $50 \mathrm{~cd}$ \\
Number of LEDs & $25(5 * 5)$ \\
LED interval & $0.02 \mathrm{~m}$ \\
Semi-angle at half power & $70^{\circ}$ \\
\hline
\end{tabular}




\subsection{Illuminance of LED Lighting}

The illuminance expresses the brightness of an illuminated surface [1]. A horizontal illuminance Ehor at a point $(\mathrm{x}, \mathrm{y})$ is given by

$$
E_{\text {hor }}=\frac{I(0) \cos ^{n}(\phi)}{R^{2} \cdot \cos (\psi)}
$$

where $\mathrm{I}(0)$ is the center luminous intensity of an LED, $\phi$ is the angle of irradiance, $\psi$ is the angle of incidence, and $\mathrm{R}$ is the distance between an LED and a detector's surface. It is assumed that an LED has a Lambertian radiation pattern according to [1]. Thus, the radiant intensity depends on the angle of irradiance $\phi . \mathrm{n}$ is the order of Lambertian emission and is expressed as the function of semi-angle at half power given below.

$$
n=-\frac{\ln 2}{\ln \left(\cos \phi_{\frac{1}{2}}^{1}\right)}
$$

Standard illuminance of lights for common office room is defined by the International Organization for Standardization (ISO). Illuminance of 300 to $1500 \mathrm{~lx}$ is required according to this standard. Figure 2 shows that sufficient illuminance can be obtained all over the room. As the minimum illuminance is $344.3 \mathrm{~lx}$ and the maximum is $456.0 \mathrm{~lx}$. Therefore, the result verifies that LED lighting devices can meet the lighting demands for indoor environment.

\section{Channel Model}

In an empty room, the light emitted from the LEDs will be reflected several times between the walls before arriving at the receiver (PD). So the calculation of the overall response which consists of direct and reflection response is more complicated. [5] presents a recursive method for calculating the impulse response containing multiple reflections. Room surfaces acting as Lambertian reflectors reflect an incident signal in all directions [5].

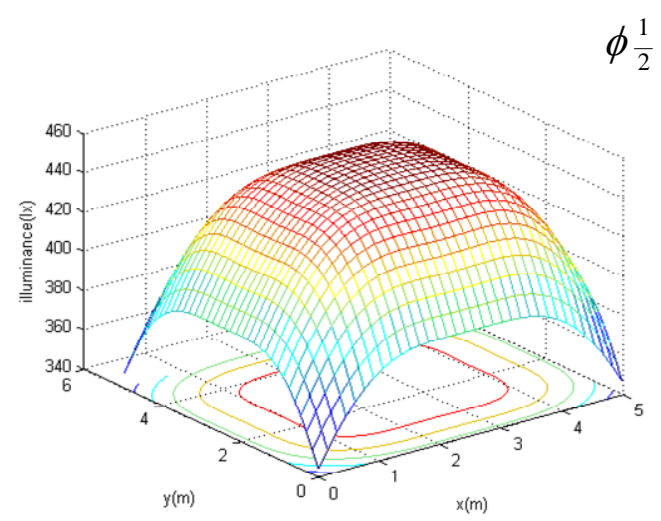

Figure 2. The distribution of the received illuminance of the floor. Min.344.3 lx, Max.456.0 lx, Ave.424.0 lx.
In an optical link, the channel DC gain is given as

$$
h(0)= \begin{cases}\frac{n+1}{2 \pi R^{2}} \cos ^{n}(\varphi) \cos (\psi) A_{R} & \theta \leq F O V \\ 0 & \text { others }\end{cases}
$$

where $A_{R}$ is the physical area of the detector in a PD. $R$ is the distance between a transmitter and a receiver, $\phi$ is the angle of irradiance, $\psi$ is the angle of incidence, $\mathrm{n}$ is the mode number of Lambertian radiation. The received optical power $P_{R}$ is derived by the transmitted optical power $P_{s}$, as follows

$$
P_{R}=h(0) \cdot P_{S}
$$

In the Figure 3, we show the distribution of received power on the floor. The average power received is 21.5 $\mathrm{dbm}$. From the formula (4), we can learn $P_{R}$ has the same distribution trend as the channel matrix $(\mathrm{H})$.

We simplified the channel model and only considered the first reflection of the light emitting from the LEDs. The more times of reflections occur, the lower received power we get. Proportion of response for each reflection compared to the total response is described in [3]. The first reflection response is given as follows [3]

$$
h(1)= \begin{cases}\frac{n+1}{2 \pi} \sum_{i=1}^{N} \frac{\rho_{i} \cos ^{n}(\varphi) \cos (\psi)}{R^{2}} h^{\prime}(0) \Delta A & 2 \theta \leq \pi \\ 0 & \text { others }\end{cases}
$$

where $\phi$ is the angle of irradiance, $\theta$ is the angle of incidence in the first reflection, $\mathrm{N}$ is the total number of elements. $\rho_{i}$ is the reflectivity at the $i$ the element. $h^{\prime}(0)$ is the response of the reflection area to the floor. $\Delta A$ is the element's area. $\Delta A$ is given by [5]

$$
\Delta t=\sqrt{\Delta A} / c
$$

where $\mathrm{c}$ is the light speed.

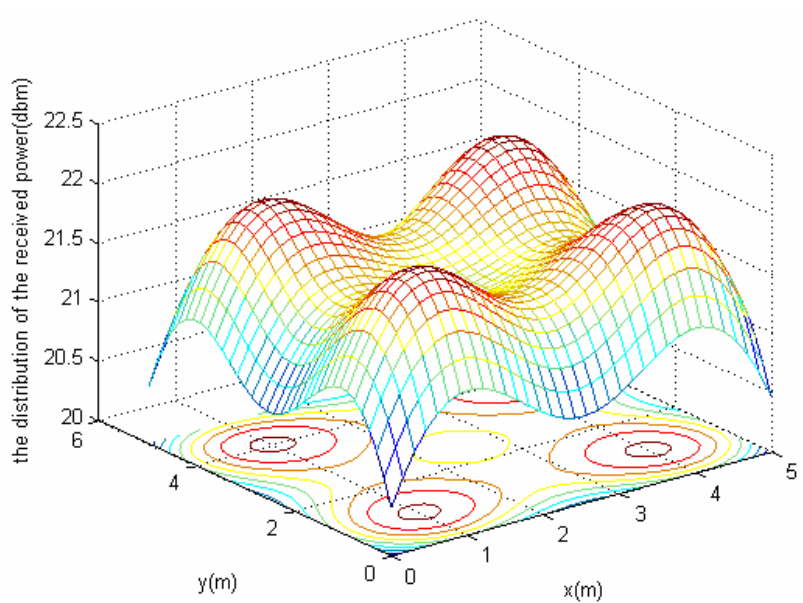

Figure 3. The distribution of received power. Min.20.4 dbm, Max.22.0 dbm, Ave.21.5 dbm. 
Figure 4 shows the distribution of impulse response with first reflection on the floor. the first reflection makes more contribution to the four boundaries on account of the limitation of FOV. The average response 3.9372e-006 is an order of magnitude lower than that of the LOS impulse response(1.1e-003). The each corner has the maximum impulse response which is 6.1279e005. It means that the reflection has little influence on the optical MIMO communication performance in this model.

The distribution of the total impulse response and LOS impulse response are showed separately in Figure $\mathbf{5}$ and Figure 6. There are four peaks just under the four LEDs arrays. It can be found that the LOS impulse response and the response including first reflection is same with the value and distribution of LOS impulse response. The maximum is 0.0013 and the average is 0.0011 .

\section{Channel Capacity}

Channel capacity expresses the maximum data rate that can be obtained by a given channel, The Shannon capacity of the MIMO system is followed by [9]

$$
C=\log _{2} \operatorname{det}\left(I+\frac{\rho}{m} H \cdot H^{+}\right) \text {bits } / \mathrm{s} / \mathrm{Hz}
$$

where $m$ is the number of transmission/receivers, $\rho$ is the average signal-to-noise ratio (SNR), $I$ is $m \times m$ identity matrix, $H$ is the normalized channel matrix, and “+” means transpose conjugate.

Table 2: Parameters for the first reflection.

\begin{tabular}{|c|c|}
\hline Parameters & values \\
\hline$\rho_{\mathrm{NORTH}}=\rho_{\mathrm{SOUTH}}=\rho_{\mathrm{EAST}}=\rho_{\mathrm{WEST}}$ & 0.8 \\
\hline Mode of Lambertian radiation (walls) & 1 \\
\hline$\Delta t$ & $0.2 \mathrm{~ns}$ \\
\hline
\end{tabular}

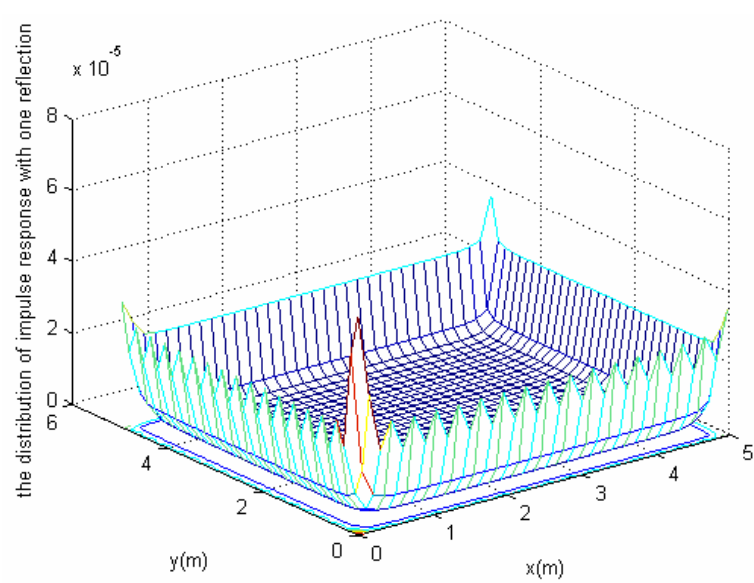

Figure 4. The distribution of impulse response with first reflection Min.8.5715e-008, Max.6.1279e-005, Ave.3.9372e006.

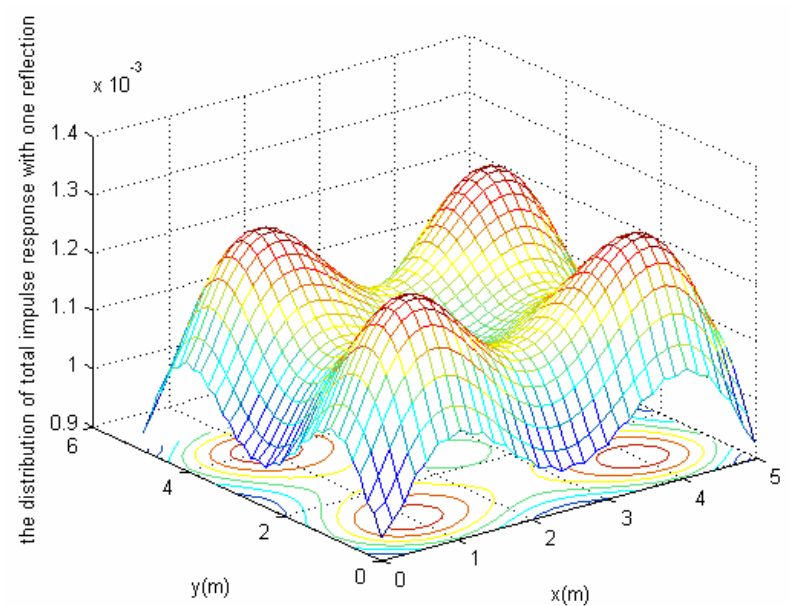

Figure 5. The distribution of total impulse response containing direct and first reflection. Min. 9.2952e-004, Max.0.0013, Ave.0.0011.

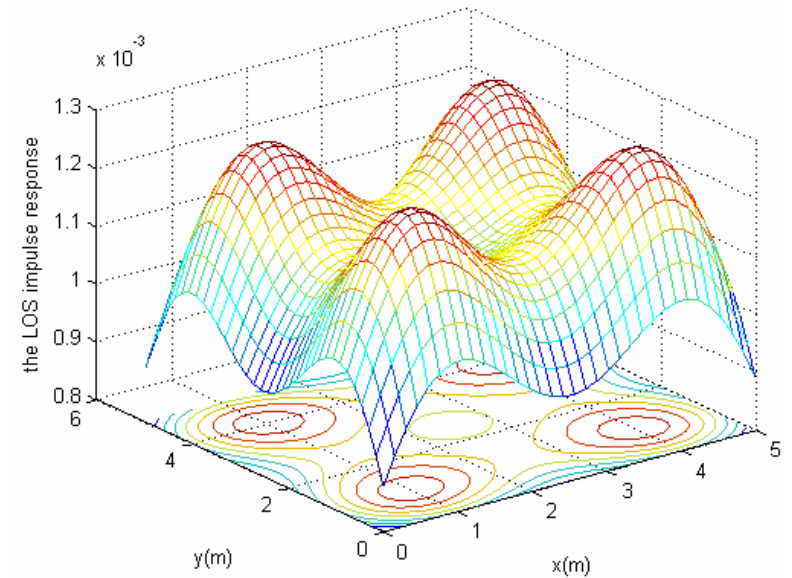

Figure 6. The distribution of LOS impulse response. Min. 8.7831e-004, Max.0.0013, Ave.0.0011.

According to the equation 7, it can be found that the channel capacity of the MIMO system depends on the channel matrix $\mathrm{H}$ (total impulse response) when average SNR $\rho$ is constant. The channel matrix $\mathrm{H}$ is determined by the configuration of the transceiver elements. Then the relationship between SNR, channel matrix $\mathrm{H}$ and capacity $\mathrm{C}$ are analyzed .

The four PDs lie in $(0.75,0.75,0),(4.25,0.75,0),(4.25$, $4.25,0),(0.75,4.25,0)$ separately in the room showed in Figure 7. Figure 8 is the relationship between the average SNR $\rho$ and the MIMO channel capacity, where the receivers are the four PDs. The simulation curve shows that the MIMO channel capacity becomes larger with the increase of $\rho$, So the high $\rho$ of the LED can result to the large capacity of the optical MIMO communication.

Figure 9 is the distribution of the capacity of one corner. The average SNR is $50 \mathrm{db}$. The capacity ranges from $2.6 \mathrm{bit} / \mathrm{s} / \mathrm{HZ}$ to $0.8 \mathrm{bit} / \mathrm{s} / \mathrm{HZ}$. It is assumed in the simula- 
tion that the four receivers are placed symmetrically in the room. So we just display the distribution of one corner. The maximum capacity shown in Figure 9 is achieved at the point just under the transmitter. In the MIMO model, the optimal location is just under each transmitter. We can achieve the maximum data rate in this location.

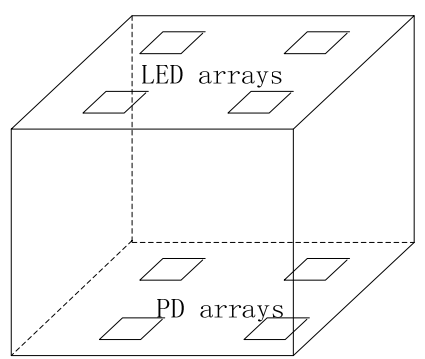

Figure 7. The distribution of four LED arrays and four PDs in the room.

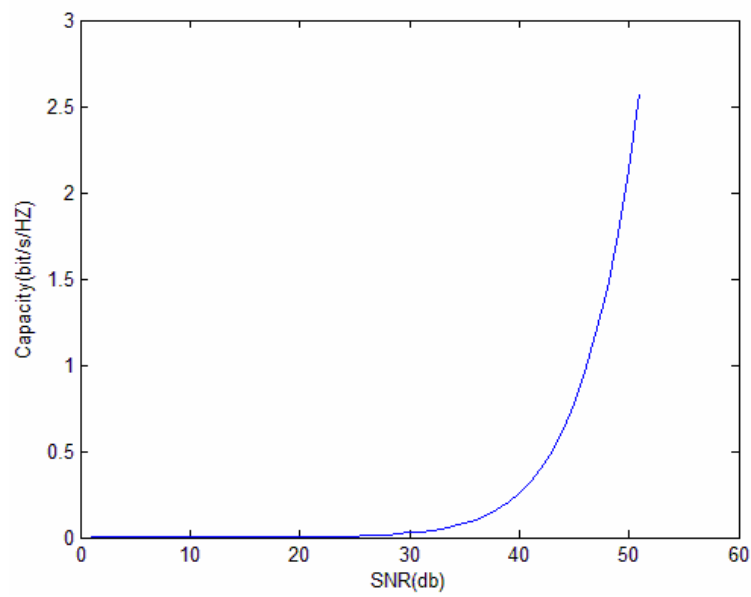

Figure 8. The MIMO channel capacity vs. average SNR. The receiver's location is $(0.75,0.75,0),(4.25,0.75,0),(4.25$, $4.25,0),(0.75,4.25,0)$.

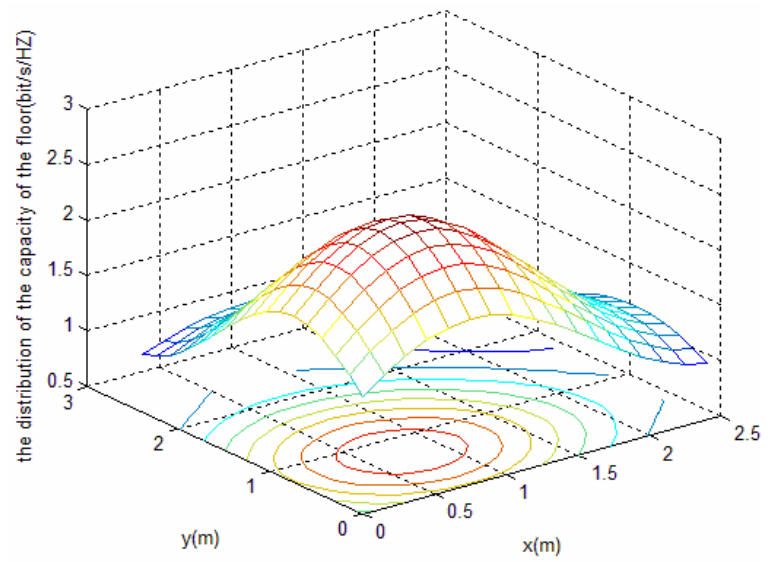

Figure 9. The distribution of the capacity of corner of the floor. The average SNR = 50db. Min.0.8 (bit/s/HZ), Max.2.6 (bit/s/HZ), Ave.1.6 (bit/s/HZ).

\section{Conclusions}

The MIMO model discussed in this paper can provide the functions including lighting and communication. We carried out the fundamental analysis for optical MIMO system which can provide theoretical basis for constructing the optimal visible light communication system. In this paper, the feasibility of visible light MIMO communication is verified by simulating the illuminance and capacity received. The effect of the parameters on the channel capacity of the MIMO system needs to be further studied in the future such as the properties of the transmitter receiver. Higher efficiency LED developed recently could largely promote the progress of visible light communications.

\section{Acknowledgements}

This work is jointly supported by NSFC (11104147), Jiangsu 973 project (BK2011027) and research project (NY211001, BJ211026).

\section{REFERENCES}

[1] T. Komine and M. Nakagawa, "Fundamental Analysis for Visible Light Communication System using LED Lights," IEEE Transaction on Consumer Electronics, Vol. 50, No.1, 2004, pp. 100-106. doi:10.1109/TCE.2004.1277847

[2] J. Vucic, C. Kottke, S. Nerreter, K. D. Langer and J. W. Walewski, "513 Mbit/s Visible Light Communications Link Based on DMT-Modulation of a White LED," Journal of Lightwave Technology, Vol.28, No.24, 2010, pp.3512-3517.

[3] D. O’Brien, R. Turnbull, H. L. Minh, G. Faulkner, O. Bouchet, P. Porcon, M. E. Tabach, E. Gueutier, M. Wolf, L. Grobe and J. H. Li, "High-Speed Optical Wireless Demonstrators: Conclusions and Future Directions," Journal of Lightwave Technology, Vol. 30, No.13, 2012, pp. 2181-2187. doi:10.1109/JLT.2012.2193874

[4] L. Zeng, D. O’Brien, H. Le-Minh, G. E. Faulkner, K. Lee, D. Jung and E. T. Won, "High Data Rate Multiple Input Multiple Output (MIMO) Optical Wireless Communications Using White LED Lighting,” IEEE Journal on Selected Areas in Communications, Vol. 27, 2009, pp. 1654-1662. doi:10.1109/JSAC.2009.091215

[5] J. R. Barry, J. M. Kahn, W. J. Krause, E. A. Lee and D. G. Messerschmitt, "Simulation of Multipath Impulse Response for Indoor Wireless Optical Channel," IEEE Journal on Selected Areas in Communications. Vol.11, No.3, 1993, pp. 367-379. doi:10.1109/49.219552

[6] J. B. Carruthers and J. M. Kahn, "Modeling of Nondirected Wireless Infrared Channels," IEEE Transaction on Communications, Vol. 45, No. 10, 1997, pp. 1260-1268.doi:10.1109/26.634690

[7] K. D. Dambul, D. O’Brien and G. Faulkner, "Indoor Optical Wireless MIMO System with an Imaging Receiver," 
IEEE Photonics Technology Letters, Vol. 23, No. 2, 2011, pp. 97-99. doi:10.1109/LPT.2010.2091627

[8] Y. A. Alqudah and M. Kavehrad, "MIMO Characterization of Indoor Wireless Optocal Link Using a Diffuse-Transmission Configuration," IEEE Transaction on Communications, Vol. 51, No. 9, 2003, pp. 1554-1560. doi:10.1109/TCOMM.2003.816945
[9] S. L. Loyka, "Channel Capacity of MIMO Architecture Using the Exponential Correlation Matrix,” IEEE Communications Letters, Vol. 5, No. 9, 2001, pp. 369-371. doi:10.1109/4234.951380

[10] X. Zhang, K. Y. C, H. Zhang and Z. Xu, "Capacity of MIMO Visible Light Communication Channels,” IEEE, WB3.4 (Contributed Oral), 2012, pp. 159-160. 